\title{
Detection of Aspergillus With Real-Time Fluorescent Quantitative PCR
}

Hui Zou', Xiangling $\mathrm{He}^{1 *}$, Yang Yang ${ }^{1}$, Yalan You ${ }^{1}$, Runying Zou ${ }^{1}$, Keke Chen ${ }^{1}$, Chengguang Zhu' ${ }^{1}$, Xin Tian ${ }^{1}$

\begin{abstract}
${ }^{1}$ Department of Hematology of Children's Medical Center, First Affiliated Hospital of Hunan Normal University (Hunan Provincial People's Hospital), No. 61, Jiefang West Road, Changsha, Hunan 410005, P.R. China.
\end{abstract}

*Corresponding author: Xiangling He. Email: 1728549500@qq.com.

\section{ABSTRACT}

Objective: To establish a real-time fluorescent quantitative PCR (FQPCR) detection system for aspergillus in order to quickly, accurately detect common aspergillus pathogens in clinically invasive fungal infections.

Methods: Standard strains of cultured fungi were used to prepare spore suspension samples with a concentration of 1,000,000 spores/ $\mathrm{mL}$. DNA samples were extracted with a one-step lysing heating method (LHM). Primers and probes for aspergillus fumigatus ( $A$. fumigatus), aspergillus flavus (A. flavus), aspergillus niger (A. niger), and aspergillus terreus (A. terreus) were designed with the Primer Premier 5.0 and Beacon Designer 7.7. The most effective primer probe was obtained after an FQ-PCR detection system screening which tested the efficiency and amplification effect for extracting aspergillus DNA via a one-step LHM, and verified specificity, repeatability, and sensitivity of the reaction system.

Results: No non-specific amplification was found. Coefficient of variation (CV) of $\mathrm{Ct}$ values in repetitive intra- and inter-assay experiments were less than $5 \%(0.37 \%-3.53 \%)$. All four aspergillum sensitivities reached at least 35 spores $/ \mathrm{mL}$ after the established reaction system was detected.

Conclusion: 1. Samples were prepared with spores cultured from aspergillus standard strains. Aspergillus DNA was extracted with an LHM method and was applied to the real-time fluorescent quantitative PCR detection. 2. Primers and probes for aspergillus fumigatus, aspergillus flavus, aspergillus niger, and aspergillus terreus were 
successfully designed and screened. Real-time FQ-PCR detection system for aspergillus was established. It was highly specific, sensitive and repeatable.

Key Words: Real-time FQ-PCR; Aspergillus; Detection

\section{INTRODUCTION}

Invasive aspergillosis (IA) is a chronic funguscaused disease caused by aspergillus. It affects the respiratory, blood, digestive, and other systems. It is the most common respiratory system fungal infection ${ }^{[1]}$. There are many species of aspergillus. Aspergillus fumigatus is the most common IA pathogen, followed by aspergillus flavus, aspergillus terrus, and aspergillus niger ${ }^{[2]}$. Children who have organ transplants such as lung or stem are highly susceptible to Aspergillus infections [AI]. Mortality can be as high as $66 \%$ and $76 \%$ depending on risk factors ${ }^{[3]}$. Children, who have a neutrophil count of less than 500 cells/mm3, large burn areas, tumors, or who have undergone long-term immuosupression or hormone therapy are susceptible to Al. Their postinfection mortality is greater than $70 \%{ }^{[4]}$.

Early diagnosis and treatment are closely associated with prognosis. Presently, the auxiliary detection method IA diagnosis includes smear microscopy, fungal culture, histopathological examination, imaging method (high resolution $\mathrm{CT}$ ), and serological method. None of these methods combined with clinical manifestations accurately or quickly detect IA. PCR technological advances have improved the speed and accuracy of Al diagnosis [5]. FQ-PCR is the most widely-used and studied method and is the most feasible.

This experiment attempted the design of the most theoretically-feasible primer probe specific for each aspergillus. It prepared spore suspension samples of aspergillus, extracted the DNA for FQPCR detection, and screened the appropriate probes. It established a real-time FQ-PCR detection system for aspergillus fumigatus, aspergillus flavus, aspergillus niger, and aspergillus terreus.

\section{MATERIALS AND MRTHODS}

\subsection{Materials}

\subsubsection{Experimental fungus strain}

The experimental fungal standard strains were aspergillus fumigatus (CGMCC3.5305), apergillus flavus (CGMCC3.5306), apergillus terreus (CGMCC3.4341), aspergillus niger (CGMCC3.4335), aspergillus nidulans (CGMCC3.3915), Candida guilliermondii (CGMCC2.1848), Candida sake (CGMCC2.1784), aspergillus clavatus (CGMCC3.5441), aspergillus versicolor (CGMCC3.5265), penicillium chrysogenum (CGMCC3.521), rhizopus arrhizus (CGMCC3.2672), mucor (CGMCC3.5007), and penicillium marneffei (PM) (CGMCC3.5831), which were purchased from China General Microbiological Culture Collection Center (CGMCC), Beijing.

Bacteria required for the experiment was limited to the following: streptococcus pneumoniae, escherichia coli, enterococcus faecium, klebsiella oxytoca, pseudomonas aeruginosa, klebsiella pneumoniae, enterobacter cloacae, staphylococcus aureus, stenotrophomonas maltophilia acinetobacter baumannii, staphylococcus epidermidis, enterobacter aerogenes, enterococcus faecalis, saphylococcus aureus, and burkholderia cepacia. They were provided by the Microbiology Department of Hunan Provincial People's Hospital, Changsha.

Hepatitis B virus (HBV), mycoplasma (MP), chlamydia psittaci (CP), human cytomegalovirus (HCMV), tubercle bacillus (TB), and Epstein Barr virus (EBV) were provided by Hunan Sansure Biotech Inc., Changsha.

Healthy control whole blood was collected from healthy volunteers at Hunan Provincial People's Hospital, Changsha.

\subsubsection{Main reagents and instruments}

Sabouraud's dextrose agar (SDA) dry powder (Qingdao Rishui Bio-technologies Co., Ltd and production number: 11193-1), E.Z.N.A. TM Fungal DNA Mini Kit (OMEGA Corporation), onestep combined heating method DNA extraction kit and one-step LHM DNA extraction kit (Hunan Sansure Biotech Inc.), Tris-HCI EDTA, Buffer, dNTP $(0.1 \mathrm{~mol} / \mathrm{L}), \mathrm{MgCl} 2(1 \mathrm{~mol} / \mathrm{L})$, and Taq enzyme (TaKaRa Biotechnology Dalian Co., Ltd.), blood/ cell/tissue genomic DNA extraction kit (Tiangen Biotech Beijing Co., Ltd.), autoclave (Beijing Xinhua Sterilization Company, and model: XGI.DMXD$0.36)$, fluorescence quantitative PCR instrument (Applied Biosystems Corporation, and model: 7500 Real Time PCR System), fluorescence quantitative PCR instrument (Agilent Technologies Co. Ltd, and model: 3000 Real Time PCR System), an common PCR instrument (Applied Biosystems Corporation, 
and model: 2720 Thermal Cycler) were used in this study.

\subsection{Methods}

\subsubsection{Inoculation and culture of aspergillus}

Bacteria in an ampoule bottle was dipped with a disposable, sterile plastic inoculating loop and planted on Sabouraud dextrose agar (SDA) plate medium using the three-spot technique. It was sealed with parafilm and incubated for 3-4 days. The medium was then inverted in a mold incubator at $37^{\circ} \mathrm{C}$. A standard-strain Petri dish was rinsed with normal saline to collect the spores after the culture of 3-4 days. Spores were counted with a hemocytometer. A suspension liquid with spore concentration of about 105 spores $/ \mathrm{mL}$ was prepared as the standard strain stock solution and refrigerated at $-70^{\circ} \mathrm{C}$ for as a standby.

\subsubsection{Extraction of aspergillus DNA}

Aspergillus DNA was extracted with a one-step combined heating method: $1 \mathrm{~mL}$ sample suspension was taken and centrifuged for $5 \mathrm{~min}$; the supernatant was removed; the sample solution was rinsed by adding $1 \mathrm{mLTE}$ buffer solution; it was centrifuged for $5 \mathrm{~min}$; the supernatant was removed; the remaining sample solution was subject to a water bath at $56^{\circ} \mathrm{C}$ for $10 \mathrm{~min} ; 100 \mu \mathrm{l}$ nucleic acid release agent and $10 \mu \mathrm{l}$ proteinase $\mathrm{K}$ was added; it was then subject to a water bath at $95^{\circ} \mathrm{C}$ for $10 \mathrm{~min}$; and the mixture was centrifuged for $5 \mathrm{~min}$ and then the supernatant was removed.

\subsubsection{Primer and probe design, and product sequencing}

Aspergillus fumigatus, aspergillus flavus, aspergillus niger, and aspergillus terreus genomic sequences were downloaded from the GeneBank of the National Center for Biotechnology Information (NCBI). Relatively-conserved sequences for four aspergillus genes were identified. Primers and FAM (Fast Auxiliary Memory) probes for each one were separately designed with the Primer Premier 5.0 and Beacon Designer 7.7. The sequences were subjected to Blast analysis via the Genebank nucleotide sequence database to detect high homologies with the nucleic acid sequences of other bacteria, virus, or human genes. Primers and probes were synthesized by Shanghai Branch of Invitrogen Corporation. The amplification products were sent to
Sangon Biotech (Shanghai) Co., Ltd. for sequencing.

2.2.4 FQ-PCR amplification aspergillus samples, primer and probe screening

Real-time FQ-PCR reaction system $(50 \mu l)$ contains PCR buffer $36 \mu \mathrm{l}, \mathrm{Mg} 2+(1 \mathrm{~mol} / \mathrm{L}) 0.2 \mu \mathrm{l}$, dNTPs $(0.1 \mathrm{~mol} / \mathrm{L}) 0.8 \mu \mathrm{l}$, Taq DNA polymerase $(2 \mathrm{U} / \mu \mathrm{l}) 2$ $\mu \mathrm{l}$, forward primers $10 \mathrm{pmmol}$, reverse primers 10 pmmol, and probes $5 \mathrm{pmmol}$, and sample DNA 10 $\mu l$. The conditions of real-time AF amplification were pre-degeneration for $5 \mathrm{~min}$ at $94{ }^{\circ} \mathrm{C}$ and a cycle of $15 \mathrm{~s}$ at $94^{\circ} \mathrm{C}$ and $30 \mathrm{~s}$ at $57^{\circ} \mathrm{C}$. Fluorescence signals were collected at the end of each of the 45 cycles performed.

\subsubsection{Aspergillus DNA samples from common PCR amplification and complete gene sequence acquisition}

Common PCR reaction system $(50 \mu \mathrm{l})$ contains PCR buffer $36 \mu \mathrm{l}, \mathrm{Mg} 2+(1 \mathrm{~mol} / \mathrm{L}) 0.2 \mu \mathrm{l}$ and $(0.1 \mathrm{~mol} / \mathrm{L})$ $0.8 \mu \mathrm{l}$, Taq DNA polymerase $(2 \mathrm{U} / \mu \mathrm{l}) 2 \mu \mathrm{l}$, forward primers $10 \mathrm{pmmol}$, reverse primers $10 \mathrm{pmmol}$, and sample DNA $10 \mu \mathrm{l}$. CPCR reaction amplification was performed as follows: pre-degeneration at $95{ }^{\circ} \mathrm{C}$ for $5 \mathrm{~min}$, degenerate at $95{ }^{\circ} \mathrm{C}$ for $30 \mathrm{~s}$, anneal at 50 ${ }^{\circ} \mathrm{C}$ for $30 \mathrm{~s}$, extend at $72{ }^{\circ} \mathrm{C}$ for $1 \mathrm{~min}$, repeat for 30 cycles, and extend at $72{ }^{\circ} \mathrm{C}$ for $7 \mathrm{~min}$.

\subsubsection{Specificity testing}

Other fungal DNA, which did not include aspergillus fumigatus, aspergillus flavus, aspergillus terreus or aspergillus niger was extracted using a onestep combined heating method. The bacteria and virus obtained were extracted using a one-step LHM nucleic acid extraction kit. The human wholeblood genome was extracted using a blood cell and tissue genomic DNA kit. These DNA samples were amplified via FQ-PCR with the primers and probes identified above and a specificity test was performed.

\subsubsection{Repetitive experiments}

Intra- and inter-assay repetitive experiments were performed. The intra-assay repetitive experiment tested the same templates and reaction systems simultaneously. The inter-assay repetitive experiment was repeated, under identical conditions using the same reaction system and the same sample over a five-day period. Suspension samples of 105-103 spores $/ \mathrm{mL}$ at three different concentration levels 
were used for the intra- and inter-assay repetitive experiments. Each sample was tested for 20 times. Intra- and inter-assay cycle thresholds (Ct) CVs were calculated with statistical software SPSS19.0.

\subsubsection{Sensitivity experiment}

A standard strain stock solution of 102 spores $/ \mathrm{mL}$ was diluted into the pre-test sample in a ratio of $1: 1,1: 2$, and 1:3. Sample DNA was extracted with the one-step combined heating method. Then the selected primers and probes were subjected to the FQ-PCR analysis to find the minimum positive-test concentrations which was the sensitivity test for this method.

\subsection{Statistical Analysis}

The mean value and standard deviations for Ct values for intra- and inter-assay repetitive experiments were calculated with SPSS19.0. CV equals the standard deviation/mean value $\times 100 \%$.

\section{RESULTS}

\subsection{Primer and probe design and screening and PCR product sequencing results}

Aspergillus fumigatus, aspergillus flavus, aspergillus niger, and aspergillus terreus genomic sequences were obtained from the GeneBank. The relatively conserved sequences for these four aspergillus genes were identified. Primers and probes for each one were separately designed with the Primer Premier 5.0 and Beacon Designer 7.7. Primer and Probe sequences were subjected to the Blast analysis to determine there were sure no significant homology between the primers and probes of four aspergillus and other gene sequences. After Blast analysis, a set of high specificity primers and probes were screened for each aspergillus. The sequence of initially-designed and synthesized primers and probes for four aspergillus appear in Table 1. Sequencing results of specific amplification products were subjected to Blast analysis. All sequences were in the target gene area where the designed primers and probes were located.

Table 1. Aspergillus primer and probe sequences

\begin{tabular}{llc}
\hline Bacteria & Sequence of Primers \& Probes & Tm value $\left({ }^{\circ} \mathbf{C}\right)$ \\
\hline \multirow{3}{*}{ A. fumigatus } & F: CCTCCAGCCAAACCTGAACTT & 60.1 \\
& P: GGCACCAGAACCTCCCGCTGAAAATG & 67.4 \\
& R: TTCGTCATTGTTAGAGCCACCT & 60.2 \\
A. flavus & P: TGGGCTGTTTTGCTGTATGGTT & 62.1 \\
& R: TGCTGAAGGACTAAAAGGACAAGG & 64.6 \\
A. terreus & F: GGACCAAGTGTAAGTCACCCAATA & 60.3 \\
& P: CAACCATCCCGGTCCGTTAGAGC & 61.9 \\
& R: ACGGCAACCAACGACGAG & 63.4 \\
A. niger & F: GCCCAACCCTGACCCAACT & 59.1 \\
& P: GATACGGCGGCCCTAGAGGATACT & 65.9 \\
\hline
\end{tabular}




\subsection{Specificity test}

In order to verify aspergillus primer and probe specificity and test for the presence, or absence, of homologous sequences among the aspergillus and between the aspergillus and other bacterial, fungal, or human genes, all of the above DNA samples were simultaneously subjected to the FQ-PCR reaction under identical conditions with the selected primers and probes. After the specificity tests for four aspergillus, no specific amplification was found. Test results were showed (Fig. 1).

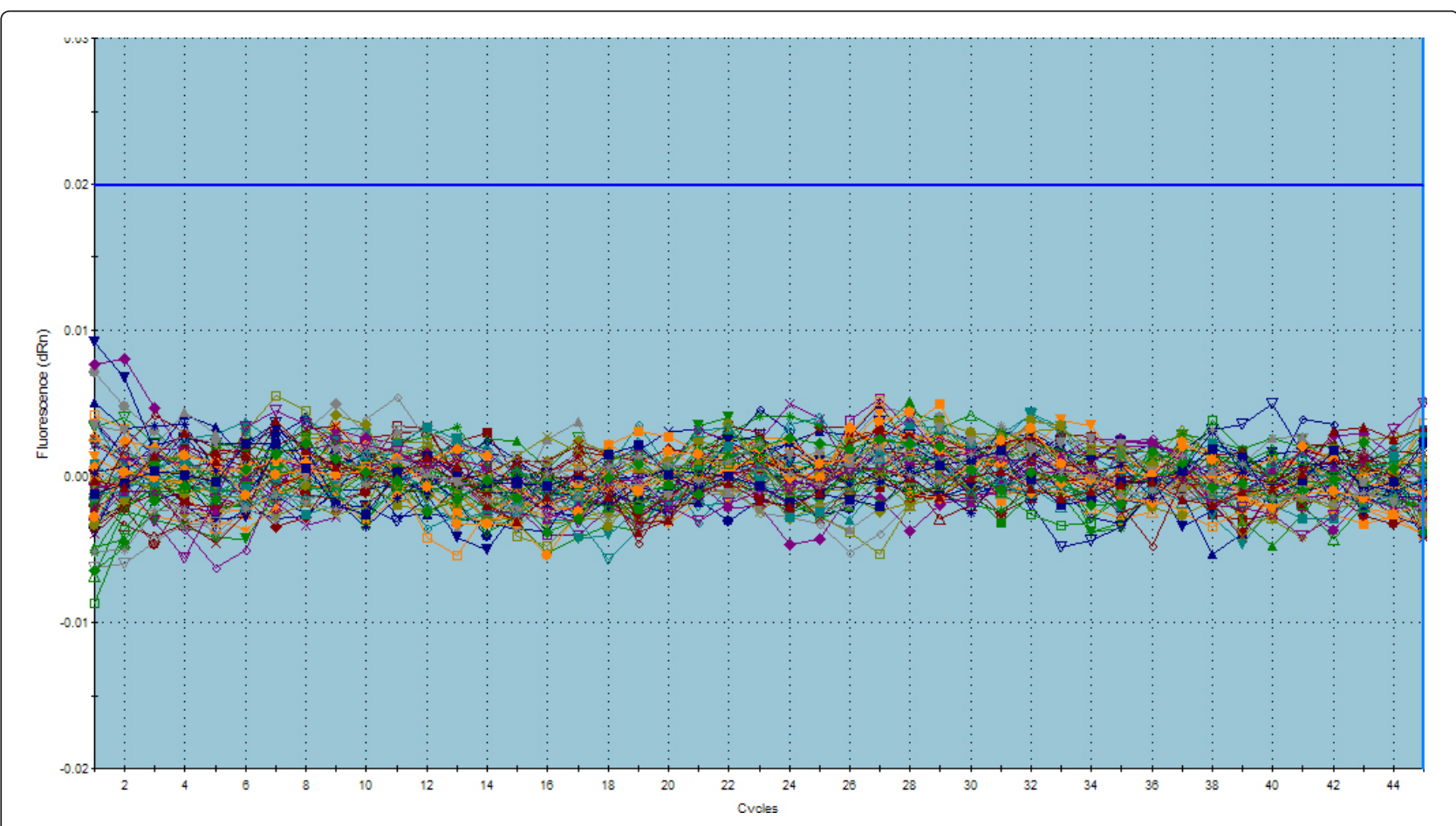

Fig. 1 Amplification curve for four aspergillus in specificity tests

No amplification curve was found for other aspergillus, fungi, bacteria, or human genes. The primers and probes had good specificity. No crossreaction was found with other fungi, bacteria, or human genes.

\subsection{Repetitive experiment}

\subsubsection{Intra-assay repetitive experiment}

DNA samples of three levels of spore concentration were extracted from aspergillus fumigatus (A. fumigatus), aspergillus flavus (A. flavus), aspergillus niger (A. niger), and aspergillus terreus (A. terreus) specimens. Twenty spores from each kind concentration were spotted and sampled simultaneously for the PCR reaction. The $\mathrm{Ct}$ value obtained was analyzed with amplification curves. The results were that the amplification curve of the same fungus obtained from the FQ-PCR detection performed under the same concentration condition coincided with that of the sample obtained from the FQ-PCR detection performed under the same concentration condition. Ct CV was less than $5 \%$ (Table 2). 
Table 2. Comparison of Ct values of four aspergillus in intra-assay repetitive experiment

\begin{tabular}{lllll}
\hline \multirow{2}{*}{ Bacteria } & Statistics & \multicolumn{3}{c}{ Concentration (Spores $/ \mathrm{mL}$ ) } \\
\cline { 3 - 5 } A. fumigatus & Mean value & 105 & $\mathbf{1 0 4}$ & $\mathbf{1 0 3}$ \\
& Standard deviation & 25.96 & 29.36 & 37.26 \\
& $\mathrm{CV}$ & 0.10 & 0.11 & 0.53 \\
A. flavus & Mean value & $0.41 \%$ & $0.37 \%$ & $1.42 \%$ \\
& Standard deviation & 26.06 & 29.38 & 37.36 \\
& CV & 0.47 & 0.11 & 0.50 \\
\multirow{3}{*}{ A. terreus } & Mean value & $1.8 \%$ & $0.37 \%$ & $1.34 \%$ \\
& Standard deviation & 26.00 & 29.51 & 37.45 \\
& CV & 0.17 & 0.38 & 0.61 \\
A. niger & Mean value & $0.65 \%$ & $1.29 \%$ & $1.63 \%$ \\
& Standard deviation & 26.27 & 29.97 & 36.33 \\
& CV & 0.49 & 0.56 & 0.69 \\
& & $1.87 \%$ & $1.87 \%$ & $1.89 \%$ \\
\hline
\end{tabular}

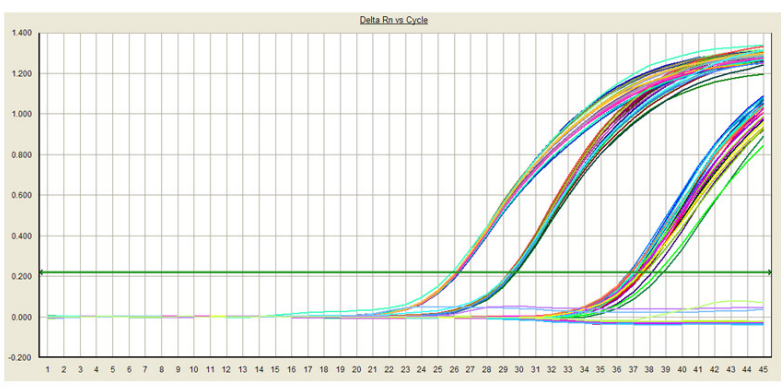

A. Fumigatus

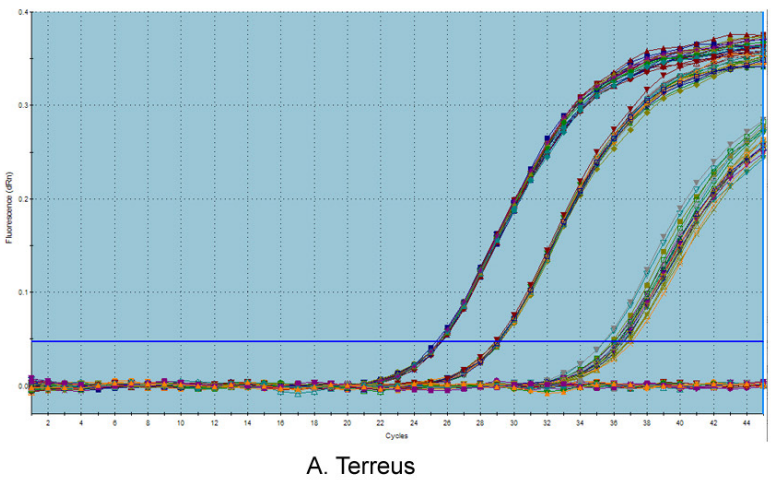

A. Terreus

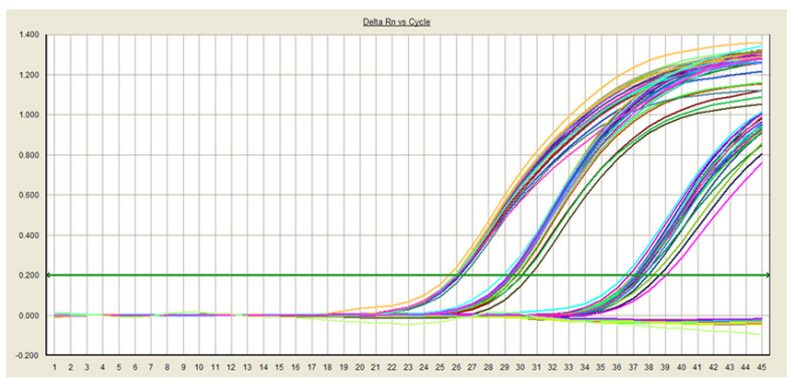

A. Flavus

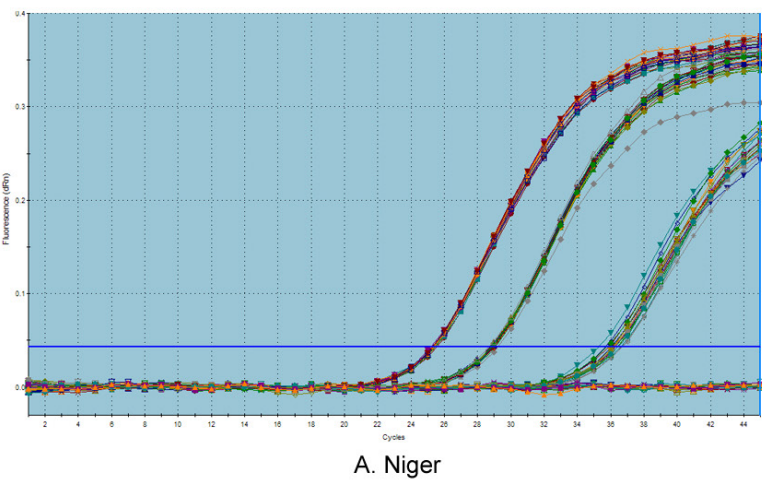

Fig. 2 Amplification curves for four aspergillus in intra-assay repetitive experiment 


\subsubsection{Inter-assay repetitive experiment}

Aspergillus fumigatus (A. fumigatus), aspergillus flavus (A. flavus), aspergillus niger (A. niger), and aspergillus terreus (A. terreus) specimuns at three different concentration levels were subjected to FQPCR detection for five consecutive days under the identical reaction conditions. Daily $\mathrm{Ct}$ mean values for the concentrations were compared. A comparison showed that mean values for CVs for the same aspergillus under the same concentration were less than $5 \%$. This indicates a good repeatability for the FQ-PCR detection system. These repeatability values appear in Table 3.

Table 3. Comparison of $\mathrm{Ct}$ values of inter-assay repetitive experiments for four aspergillus

\begin{tabular}{|c|c|c|c|c|}
\hline \multirow{2}{*}{ Bacteria } & \multirow{2}{*}{ Statistics } & \multicolumn{3}{|c|}{ Concentration (Spores $/ \mathrm{mL}$ ) } \\
\hline & & 105 & 104 & 103 \\
\hline \multirow[t]{3}{*}{ A. fumigatus } & Mean value & 25.87 & 30.36 & 36.24 \\
\hline & Standard deviation & 0.62 & 0.86 & 1.28 \\
\hline & CV & $2.39 \%$ & $2.83 \%$ & $3.53 \%$ \\
\hline \multirow[t]{3}{*}{ A. flavus } & Mean value & 26.33 & 29.88 & 36.12 \\
\hline & Standard deviation & 0.14 & 0.31 & 0.40 \\
\hline & CV & $0.53 \%$ & $1.04 \%$ & $1.11 \%$ \\
\hline \multirow[t]{3}{*}{ A. terreus } & Mean value & 26.23 & 29.93 & 36.51 \\
\hline & Standard deviation & 0.40 & 0.18 & 0.40 \\
\hline & CV & $1.52 \%$ & $0.60 \%$ & $1.09 \%$ \\
\hline \multirow[t]{3}{*}{ A. niger } & Mean value & 25.90 & 30.11 & 36.14 \\
\hline & Standard deviation & 0.56 & 0.44 & 0.59 \\
\hline & CV & $2.16 \%$ & $1.46 \%$ & $1.63 \%$ \\
\hline
\end{tabular}

The amplification in each assay reaction for the intra- and inter-assay repetitive experiments for four aspergillus, along with the amplification curves of the inter- and inter-assay repetitive experiments under the same concentration coincide. All calculated $\mathrm{Ct}$ values for CVs of amplification curves of inter- and inter- assay repetitive experiments were less than 5 $\%(0.37 \%-3.53 \%)$. This indicates that four reaction systems were stable and reproducible.

\subsection{Sensitivity experiment}

Amplification curves were easily observed in the sample size of $103 \mathrm{spores} / \mathrm{mL}$ in the repetitive tests. The samples of four aspergillus were diluted 1:1, 1:2, and $1: 3$ by taking a specimen of $102 \mathrm{spores} / \mathrm{mL}$ as the basic specimen. This was done in order to detect any lower detection limits of the PCR detection system. Diluted samples of each batch were tested for 20 times. A diluted sample served as the pre-test sample for the FQ-PCR detection. Detection results are shown (Fig. 3). 


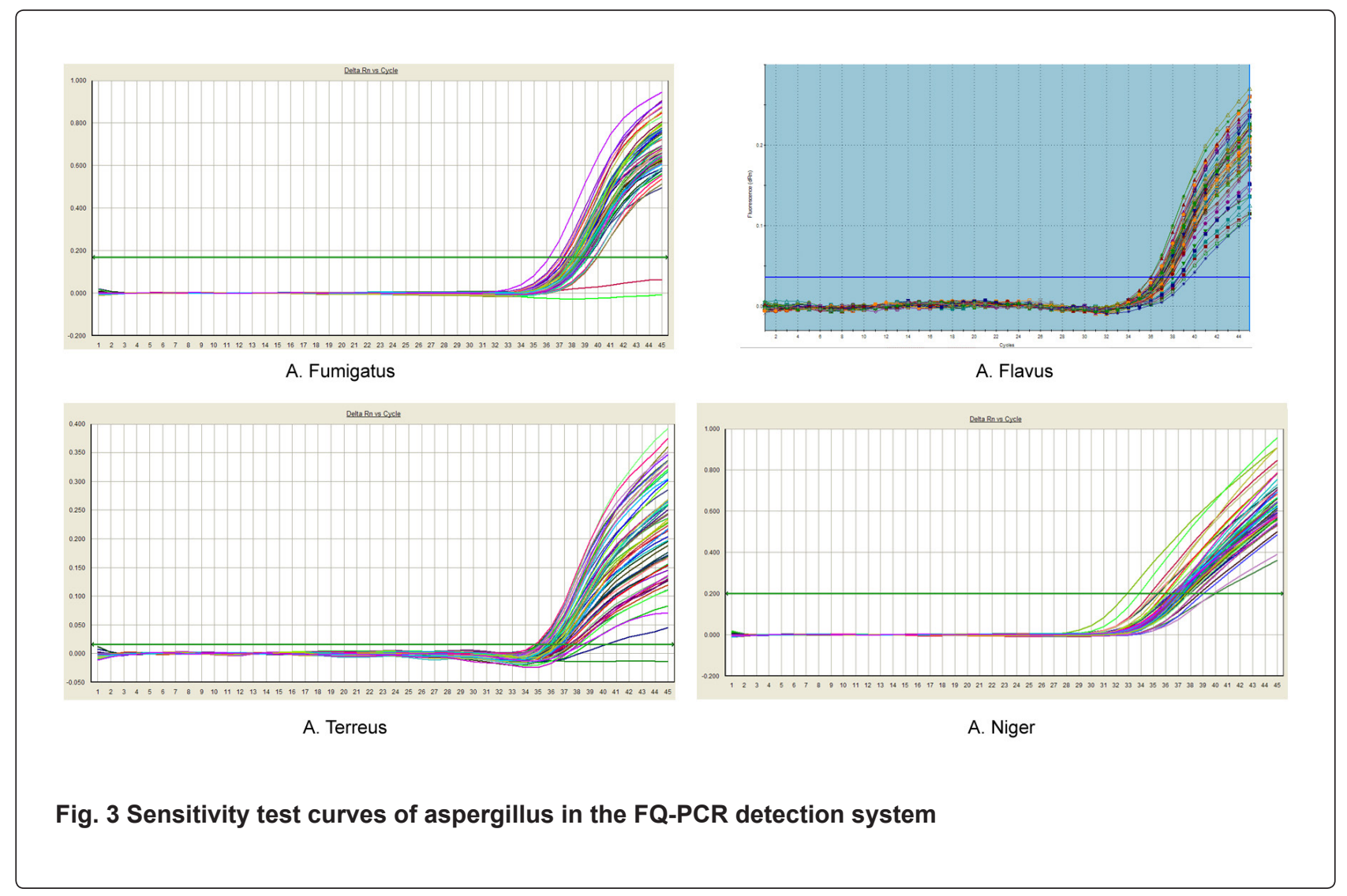

Fig. 3 shows that the amplification curves were observed for all pre-test samples of four aspergillus in the FQ-PCR detection system which was diluted at the ratios of $1: 1,1: 2$, and $1: 3$ based on the basic sample of 102 spores/mL concentration. Amplification ratios of repeated samples for each diluted concentration was greater than 95 \%. A 102 spores $/ \mathrm{mL}$ concentration of the pre-test sample diluted at a ratio of $1: 3$ from the suspension liquid can be used as the minimum detection concentration detection with sensitivity reaching approximately 35 spores $/ \mathrm{mL}$.

\section{DISCUSSION}

Primer and probe specificity are the limiting factors in establishing an FQ-PCR detection system for aspergillus and extraction of aspergillus DNA. FQPCR primers and probes select highly-conserved sequences of aspergillus as amplification target sequences to prevent high homologies with other bacterial, fungal, and human genes. The ITS sequence was the most highly conserved sequence for most fungi including aspergillus. Fungal species can be identified when high-specificity, designed primers and probes contain the ITS sequence, particularly, both ITS1 and ITS2.

Many studies have reported on the design of primers and probes for real-time FQ-PCR with this sequence ${ }^{[6-8]}$. In the instant study, primers and probes were designed using the ITS sequences. Problems for these designed probes were encountered. They were not conservative, the 5' end was $G$, and the primer Tm value failed to meet certain requirements were three instances.

Some sequences of aspergillus fumigatus eas $\mathrm{L}$, aspergillus flavus afIE, aspergillus terreus strain CBMAI 0193 reducing polyketide synthase, and aspergillus niger alpha-1,3-glucan synthase, genes were chosen as the amplification target sequences ${ }^{[9-12]}$. The conserved sequences of four aspergillus genes were searched in Genebank (NCBI) with FQ$\mathrm{PCR}$ and TaqMan probe design principles to design the primers and TaqMan probes.

The primers and probes were synthesized 
for FQ-PCR detection to screen and obtain the primers and probes having high specificity and good curves. The FQ-PCR primers and four aspergillus DNAs served as the substrate for a common PCR detection. They were sent for PCR amplification product sequencing. Homologies between products and four aspergillus were compared. The results showed a higher homology (up to $90 \%$ ) between amplification products and four aspergillus gene fragments. This indicates no non-specific amplification product. The results of FQ-PCR detection system specificity, sensitivity, and repeatability testing show a high specificity, no cross-reaction with other fungal, bacterial or human genes. It also returns sensitivity with a concentration of up to 35 spores $/ \mathrm{mL}$, and a repeatability. CVs Ct values obtained from intra- and inter-assay repetitive experiments with SPSS 19.0 were less than $5 \%(0.37$ $\%-3.53 \%)$. This complies with National Standard Nucleic Acids Amplification Test Reagents (Kits) (YYI $\mathrm{T}$ 1182-2010) requirements approved by the CFDA on December 27, 2010 for implementation on June 1,2012 . This study used a DNA extraction method suitable for liquid-type specimens and established an aspergillus real-time FQ-PCR detection system with a high specificity and a good sensitivity and reproducibility. This provides a reference for molecular biology methods for the clinical diagnosis of aspergillus-caused illnesses.

This one-step combined heating method can be applied in liquid-type specimens. The experimental results obtained have been verified through a standard strain suspension with a good amplification curve in QD-PCR system. Other factors are present, such as enzymes, inorganic salts, other plasma components there are the factors influencing the buffer concentration and Taq enzyme activity in the PCR reaction system. This may result in a low mycelium and spore concentration collected in the alveolar lavage fluid. There are also a variety of infectious viruses, bacteria and other inhibitory factors which have effects that need accounting for. There may be other unknown substances that impact on the process. Therefore, there remains a level of uncertainty in the DNA extraction of clinical specimens of plasma and alveolar lavage. False negative amplification results may also occur. More clinical samples are needed for verification.

The primers and probes for the FQ-PCR detection system in this study were targeted at $A$. fumigatus, $A$. flavus, $A$. terreus, and $A$. niger. These are a wide range of common clinical fungi, but were tested separately. The same clinical suspect specimen should be tested simultaneously with four detection systems which are cumbersome. There are many species of aspergillus and each has also a variety of subspecies. There is no certainty that the specificity of a primer or probe adapts to other subspecies. It should be verified by a large number of samples to be collected. The real-time FQ-PCR detection system tested in this study for the diagnosis of clinical aspergillosis needs further testing using more clinical specimens.

In summary, as more and more studies of FQ-PCR technology in the diagnosis of invasive aspergillosis has been achieved even though this experiment had limitations. It is expected to open up a new diagnostic pathway for rapid, sensitive and specific diagnosis of the invasive aspergillosis.

\section{ACKNOWLEDGEMENT}

We acknowledge the financial support from the Science and technology program of Department of Science and Technology of Hunan Province (Program No.: 2013FJ6028).

\section{REFERENCES}

1. Arvanitis M, Anagnostou T, Mylonakis E. Galactomannan and polymerase chain reactionbased screening for invasive aspergillosis among high-risk hematology patients: A diagnostic Meta-analysis. Clin Infect Dis. 2015; 61(8): 1263.

2. Kwon-Chung KJ, Sugui JA. A spergillus fumigatus - what makesthe species a ubiquitous human fungal pathogen? PLoS Pathog. 2013; 9 (12): e1003743.

3. Geltner C, Lass-Florl C. Invasive pulmonary aspergillosis in organ transplants - focus on lung transplants. Respir Investig. 2016; 54(2): 76-84.

4. Camargo JF, Husain S. Immune correlates of protection in human invasive aspergillosis. Clin Infect Dis. 2014; 59(4): 569-577.

5. Rogers TR, Morton CO, Springer J, Conneally E, Heinz W, Kenny C, Frost S, Einsele H, Loeffler J. Combined real-time PCR and galactomannan surveillance improves diagnosis of invasive aspergillosis in high risk patients with haematological malignancies. $\mathrm{Br} \mathrm{J}$ Haematol. 2013; 161: 517-524. 
6. Rittenour WR, Ciaccio CE, Barnes CS, Kashon ML, Lemons AR, Beezhold DH, Green BJ. Internal transcribed spacer rRNA gene sequencing analysis of fungal diversity in Kansas City indoor environments. Envir Sci Process Impacts. 2014; 16(1): 33-43.

7. Tan H, Cao L. Fungal diversity in sheep (Ovis aries) and cattle (Bos taurus) feces assessed by comparison of $18 \mathrm{~S}, 28 \mathrm{~S}$ and ITS ribosomal regions. Ann Microbiol. 2014; 64(3): 1423-1427.

8. Romanelli AM, Fu J, Herrera ML, Wickes BL. A universal DNA extraction and PCR amplification method for fungal rDNA sequence-based identification. Mycoses. 2014; 57(10): 612-622.

9. Robinson SL, Panaccione DG. Chemotypic and genotypic diversity in the ergot alkaloid pathway of Aspergillus fumigatus. Mycologia. 2012; 104(4): 804-812.
10. Damveld RA, Arentshorst M, Franken A, vanKuyk PA, Klis FM, van den Hondel CA, Ram AF. The Aspergillus niger MADS-box transcription factor RImA is required for cell wall reinforcement in response to cell wall stress. Mol Microbiol. 2005; 58(1): 305-319.

11. Ehrlich KC, Chang PK, Scharfenstein LL Jr, Cary JW, Crawford JM, Townsend CA. Absence of the aflatoxin biosynthesis gene, norA, allows accumulation of deoxyaflatoxin B1 in Aspergillus flavus cultures. FEMS Microbiol Lett. 2010; 305(1): 65-70.

12. $\mathrm{Xu} Y$, Espinosa-Artiles $P$, Schubert $V, X u Y M$, Zhang W, Lin M, Gunatilaka AA, Süssmuth R, Molnár I. Characterization of the biosynthetic genes for 10, 11-dehydrocurvularin, a heat shock response-modulating anticancer fungal polyketide from aspergillus terreus. Appl Environ Microbiol. 2013; 79(6): 2038-2047. 